Topiques, études satoriennes

Topoï Studies, Journal of the SATOR

\title{
À la table d'Apollon : de l'abondance à la modération dans Francion
}

\section{Martine Debaisieux}

Volume 5, 2021

Le manger et le boire dans la fiction narrative

URI: https://id.erudit.org/iderudit/1081525ar

DOI: https://doi.org/10.7202/1081525ar

See table of contents

Publisher(s)

SATOR, Société d'Analyse de la Topique Romanesque d'Ancien Régime

ISSN

2369-4831 (digital)

Explore this journal

Cite this article

Debaisieux, M. (2021). À la table d'Apollon : de l'abondance à la modération dans Francion. Topiques, études satoriennes / Topoï Studies, Journal of the SATOR, 5, 86-104. https://doi.org/10.7202/1081525ar
Article abstract

The first edition of the Histoire comique de Francion by Charles Sorel (1623) contains numerous references to the eating and drinking. My study examines narrative sequences focused on a fluctuation between deprivation and abundance, frustration and jouissance. In addition to the domain of food, I consider allusions to sexuality and knowledge, insofar as they share the same narrative paradigm, and shed light on each other. This analysis also shows how Sorel relates ambivalent references to food to some of his moral claims, expressed through subversive uses of the tradition of comic fiction. The four books added for the 1626 and 1633 editions of Francion can be perceived as a wavering attempt to displace Bacchus, who presides over the protagonist's birth and is emblematic of the various immoderations in the first edition.
This document is protected by copyright law. Use of the services of Érudit (including reproduction) is subject to its terms and conditions, which can be viewed online.

https://apropos.erudit.org/en/users/policy-on-use/ 


\title{
À la table d'Apollon : \\ de l'abondance à la modération dans Francion
}

\author{
Martine Debaisieux \\ University of Wisconsin-Madison
}

Hortensius estoit de ceux qui aimoient les sentences que l'on trouvoit escrites au Temple d'Apollon, et principalement il estimoit celle cy, Ne quid nimis, laquelle il avoit escrite au dessus de la porte de sa cuisine, pour faire voir qu'il n'entendoit pas que l'on mist rien de trop aux banquets que l'on y appresteroit ${ }^{1}$.

La première édition de l'Histoire comique de Francion de Charles Sorel (1623) accorde une place privilégiée aux références au domaine alimentaire, tout en exploitant les métaphores qui lui sont associées. Par cette récurrence topique et par la prédilection de son champ lexical, elle se distingue des quatre livres ajoutés aux deuxième et troisième éditions, trois ans et dix ans plus tard. Dans les remaniements de son histoire comique et dans ses ajouts, Sorel fait preuve d'une relative sobriété à l'égard de la topique du boire et du manger ; cet élément de contraste s'expliquerait en partie lorsque l'on tient compte de l'ambiguité de plusieurs épisodes de l'édition initiale. Je propose d'examiner la configuration de séquences narratives axées sur une fluctuation entre privation et abondance, frustration et jouissance; ma lecture porte essentiellement sur le registre alimentaire, mais touche également à celui de la sexualité et de la connaissance, dans la mesure où ils s'inscrivent dans le même paradigme narratif, et s'éclairent réciproquement. En considérant l'agencement de la topique du manger et du boire dans Francion et ses implications pour l'interprétation du texte, mon approche met en évidence un déroulement narratif qui se joue de la chronologie pour s'articuler sur un jeu de répétitions et de contrastes entre différentes séquences.

\footnotetext{
${ }^{1}$ Histoire comique de Francion, éd. Antoine Adam, 1958, p. 1278. Mes citations se réfèrent à cette édition étant donné que cette analyse porte majoritairement sur la première version du texte. En revanche, cet exergue correspond à un ajout de l'édition de 1633 (en appendice dans l'édition d'A. Adam).
}

Topiques, Études satoriennes, 5, 2020, Le Boire et le manger, https://journals.uvic.ca/index.php/sator/index 
La représentation des scénarios alimentaires dans Francion doit être envisagée avant tout par rapport à la position subversive de Sorel vis-à-vis de ses modèles littéraires ; mais une tentative de situer l'ambiguïté récurrente du discours et de la narration ne peut complètement ignorer d'autres facteurs tels que la double visée de sa carrière d'écrivain, sa tentative de se protéger de la censure, et encore moins, le penchant mystificateur et ludique qu'il laisse paraître dans la composition de son histoire comique. En premier lieu, les variantes du motif d'ingurgitation exploitées dans Francion se rapprochent de la tradition carnavalesque qui, selon Mikhaïl Bakhtine, présente les pulsions du « corps grotesque » sur le mode du débordement et de l'excès. Ma lecture rejoint et nuance la perspective du critique qui perçoit dans les histoires comiques de Sorel un carnavalesque « au stade de la transition et de la réinterprétation ${ }^{2}$ ». Dans Francion, le caractère « joyeux » et « triomphant» de l'absorption de nourriture, tel qu'on le trouve chez Rabelais, se voit détourné, alors que le texte s'inspire encore de la topique de la fête et des banquets. Les tendances de la culture populaire sont en effet contaminées par une tradition satirique, fondée sur un rire moqueur ; en conséquence, l'excès du boire et du manger se voit réduit en gloutonnerie et ivresse, principalement à travers le portrait de personnages stéréotypes ${ }^{3}$.

L'ambivalence parfois méfiante de l'alimentaire dans Francion ne peut être séparée de la visée morale de Sorel, qui vient se greffer de manière parfois déroutante sur une tradition comique de nature hybride ${ }^{4}$. On remarque ainsi au niveau discursif - en particulier à travers les propos du protagoniste dans la première édition - l'expression fluctuante d'une apologie de la modération qui anticipe les principes de la «morale practique » que Sorel expose dans son œuvre savante. Pour nous en tenir au champ alimentaire, évoquons par exemple une remarque de De la perfection de l'homme : « [La prudence et la volonté] reigleront ensemble tout ce qui concerne le corps, nous empeschant de le nourrir par excez \& de contenter ses appetits desordonnez $z^{5} »$.

\footnotetext{
${ }^{2}$ Mikhaïl Bakhtine, L'œuvre de François Rabelais et la culture populaire au Moyen Age et sous la Renaissance, 1970, p. 110. Le critique soutient que les images du «manger » et de la beuverie telles qu'on les trouvent chez Rabelais reflètent la conception de l'homme qui « déguste le monde, sent le goût du monde, l'introduit dans son corps, en fait une partie de soi » (p. 280). Voir Robin Howells, Carnival to Classicism : The Comic Novels of Charles Sorel, 1989 ; Francis Assaf, «Francion, une étude carnavalesque », 2001, pp. 85-95.

${ }^{3}$ Au personnage du pédant glouton Hortensius se substitue celui de Gastrimargue dans Polyandre, troisième histoire comique de Sorel (1649). Notons aussi la parenté entre Hortensius et le personnage de Sydias dans Première journée (1623) de Théophile de Viau.

${ }^{4}$ Voir Richard Hogdson, «De la 'comédie humaine' à la 'perfection de l'homme' : Charles Sorel moraliste », 2006, pp. 19-30. Pour une interprétation du cheminement de Sorel entre Francion et La Science Universelle, voir Fausta Garavini, La Maison des Jeux, 1998, pp. 123-27.

${ }^{5}$ Charles Sorel, De la Perfection de l'homme, 1655, p. 3.
}

Topiques, Études satoriennes, 5, 2020, Le Boire et le manger, https://journals.uvic.ca/index.php/sator/index 
Par rapport à l'instabilité de la signification que produisent les contradictions de la première édition aussi bien que la juxtaposition de ses remaniements, il convient de rappeler également que Francion est composé au moment du procès de Théophile de Viau, que fréquentait Sorel. Certaines incohérences apparentes peuvent être envisagées dans la perspective d'une stratégie de dissimulation vis-à-vis de la censure ${ }^{6}$. Dans ce sens, notons que dans La Doctrine curieuse des beaux esprits de ce temps, le Père Garasse, le principal accusateur de Théophile, associe de manière insistante les libertins, « confrères de la bouteille », aux excès alimentaires ${ }^{7}$.

Ce bref aperçu justifie une démarche prudente dans l'interprétation de la topique alimentaire, face à la signification glissante de 1'histoire comique ${ }^{8}$. En guise d' « avant-goût», on peut s'interroger par exemple sur l'intention de Sorel dans un ajout à la dernière édition (placé ici en exergue). Lorsqu'il introduit la figure d'Apollon en choisissant la sentence « Neme quid nimis » (rien de trop) comme prescription pour la cuisine du maître de collège Hortensius, l'auteur vise-til toujours à se moquer de la parcimonie intéressée du pédant ? Ou cherche-t-il cette fois à détrôner Bacchus, qui préside à la naissance de son protagoniste et aux divers débordements qui font, pour nous, la saveur de l'histoire comique initiale?

\section{Déboires d'un nourrisson}

Francion naît le jour de la fête des Rois, date qui marque l'ouverture du carnaval. Alors qu'elle boit « aux bonnes graces de tous ses subjets d'une soirée», sa mère, « Reyne de la feve », est interrompue par une « petite » douleur suivie de manière bien expéditive de son accouchement. A la suite d'une allusion plaisante à l'intronisation qu'il peut espérer lui-même ${ }^{9}$, le narrateur précise : «L'on beut si plantureusement a ma santé par tout le logis qu'il y parut bien aux tonneaux

\footnotetext{
${ }^{6}$ Voir Jole Morgante, «La réécriture de la première partie du Francion : techniques d'écriture libertine », 2001, pp. 14-30 ; Martine Debaisieux, «Francion ou la libre pensée travestie », 1988, pp. 191-99.

${ }^{7}$ Louise Godard de Donville élabore ce rapprochement dans Le Libertin des origines à 1665 : un produit des apologètes, 1989, pp. 140-44. Voir aussi François Friche, Entre Terre et Ciel : Romans comiques et mystère de l'Incarnation, 2017, pp. 200-201.

${ }^{8}$ Martine Debaisieux met en évidence les enjeux de la composition de ce «texte protéiforme » qui défie toute interprétation univoque (Le Procès du roman : écriture et contrefaçon chez Charles Sorel, 1989). Patrick Dandrey analyse plus précisément la dynamique de réécriture du texte, et en particulier sa palinodie déroutante (Le premier Francion de Charles Sorel, ou le « jeu du roman », 2001).

${ }^{9}$ « Elle sentit une petite douleur qui la contraignit de se jetter sur un lict, où elle ne fut pas si tost, qu'elle accoucha de moy, sans sage femme [...]. Ainsi je nasquis Dauphin, et ne sçay quand ce sera que je me verray la couronne Royalle sur la teste » (p. 164). Le phénomène de couronnement et du découronnement domine le registre carnavalesque défini par Bahktine (p. 161).
}

Topiques, Études satoriennes, 5, 2020, Le Boire et le manger, https://journals.uvic.ca/index.php/sator/index 
de nostre cave » (p. 164). Cette première évocation de l'ingestion implique la dichotomie plein/vide qui s'insinue dans plusieurs épisodes de l'histoire comique.

Certes, cette atmosphère festive où le vin coule en abondance rejoindrait les circonstances de la venue au monde de Gargantua. Cependant, à la différence du personnage rabelaisien, le rapport de Francion à la nourriture dès son plus jeune âge est marqué par le manque et la frustration, liés à l'épuisement potentiel des ressources alimentaires ou à leur inaccessibilité ${ }^{10}$. Le premier indice ressort de l'évocation de son allaitement, dans lequel l'auteur prend ses distances de la tradition comique pour entrer dans le registre plus « réaliste » de la vie privée : « ma mere n’estant pas en assez bonne disposition a son advis, se disposa de me nourrir et de me bailler à une femme d'un village prochain pour me donner a teter » (p. 164). Cette référence ambiguë à l'attitude maternelle se voit rectifiée dans l'édition de 1633 : «ma mere n’estant pas en assez bonne disposition a son advis, pour estre nourrice, me bailla a une femme du village prochain [...] ». La mention de cette « disposition », nuancée de manière suspecte («a son advis »), fait l'objet d'un commentaire : «je ne veux pas m'arrester à juger si elle fit bien d'endurer que je prisse du laict d'une autre qu'elle » (p. 164). Cette prétérition est particulièrement chargée si l'on considère la réflexion morale et médicale sur la question de l'allaitement à l'époque; de fait, le narrateur poursuit en laissant entendre que lors d'une telle substitution du sein nourricier, les « humeurs » $\mathrm{du}$ « vulgaire » sont susceptibles d'être transmises à l'enfant ${ }^{11}$.

Le récit enchaîne directement sur un autre incident avilissant, que le narrateur situe peu après son sevrage ${ }^{12}$. Dans cette seconde déception des attentes alimentaires de Francion, c'est le « rituel de la bouillie » - où la mère tient un rôle essentiel à l'époque - qui se voit perverti ${ }^{13}$.

\footnotetext{
${ }^{10}$ Michel Jeanneret remarque à propos du rapport du jeune Gargantua à la nourriture : « il naît dans la profusion d'un festin et, toujours affamé, toujours altéré, percevra d'abord le monde comme la source intarissable de ses plaisirs [...]. Rien ne bride l'appétit de l'enfant » (Des Mets et des Mots, 1987, p. 71).

${ }^{11}$ Jacques Gélis précise : «La pratique est généralement déconseillée, voire condamnée par les moralistes : il est dangereux pour un jeune enfant non encore « terminé » d'être nourri d'un " lait mercenaire »; et puisqu'il est admis que «nourriture passe nature », n'est-ce pas son identité même qui risque d'être affectée par une "transfusion » concernant tant le corps que l'esprit » (Histoire de la vie privée, 1986, pp. 319-21). Jean-Louis Flandrin, quant à lui, cite Examen de la théologie morale de Fernand De Moure (1638) : « [Les mères] pèchent lorsqu'elles refusent pendant trois ans la nourriture à leurs enfants, le pouvant faire " "Alimentation et médecine. Histoire de l'alimentation occidentale », 2003, p. 9). Sorel soutient une opinion similaire dans La Science Universelle.

${ }^{12}$ Flandrin remarque : «Du haut Moyen Age aux temps modernes les moralistes prescrivaient aux mères de nourrir [les enfants] jusqu'à l'âge de trois ans » (op. cit., p. 9).

${ }^{13}$ Flandrin explique que le « rituel de la bouillie » à l'époque impliquait le «passage par la bouche de la mère » avant que ne soit présenté à l'enfant le « doigt nourricier ». "Le rôle de la salive prolonge, comme l'allaitement au sein, la relation nourricière étroite entre la mère et l'enfant qui existait avant la naissance » (op. cit., p. 11).
}

Topiques, Études satoriennes, 5, 2020, Le Boire et le manger, https://journals.uvic.ca/index.php/sator/index 
S'étant introduit subrepticement chez Francion, un « Maistre singe », nourrit « secrettement » par un voisin laboureur, remplace cette fois la mère absente pour donner à manger à l'enfant, alors que la servante qui a fait chauffer la bouillie n'est pas non plus présente pour remplir sa fonction de substitut (on apprend que l'une est à la messe ; l'autre, à la cour) : « Ayant veu, pensez, d'autres fois donner de la bouillie aux enfans, [le singe] prit un peu de la mienne et m'en vint barbouiller tout le visage »(p. 165). Francion a donc de nouveau affaire à un intermédiaire nourricier « vulgaire » et de surcroît, tout à fait inapte ; le visage couvert de la nourriture qui lui était destinée, l'enfant - à la fois tantalisé et terrorisé ${ }^{14}$ - reste sur sa faim. Le rapport perverti du protagoniste à la nourriture et la victimisation qui en résulte annoncent des motifs essentiels de l'épisode du rêve où se réfracte, comme nous le verrons, l'image de la « mauvaise mère ${ }^{15} »$.

Au cours de l'enquête visant à révéler l'identité de l'imitateur maladroit que Francion prend pour un petit garçon, il sera aussi question de nourriture dans la perspective, cette fois, de convoitise frauduleuse. En premier lieu, le récit expose le maraudage d'un paysan cherchant à « desrober [1] es fruicts » du père de Francion qui, après lui avoir assené « deux ou trois coups de pieds au cul », le menace d'être « mis en justice » s'il retourne à sa « premiere faute » (p. 167) ${ }^{16}$. La fin de l'épisode se focalise sur le larcin de quelques pigeons par les serviteurs qui s'apprêtent à «faire ripailles aux despens de leur maistre» (p. 168); ils s'assurent la complicité du jeune Francion en profitant de sa naïveté et en incitant sa gourmandise. Cette configuration narrative plus traditionnelle ${ }^{17}$ se retrouve dans l'épisode du collège sur lequel enchaîne le narrateur. Il limite en effet explicitement les références à son « bas âge » aux deux épisodes axés sur sa nutrition pour «monte[r] un peu plus haut » (p. 169).

\section{Tantale au collège}

Francion quitte le domaine familial pour être mis « en pension » au collège, où il sera pris en charge par un « maistre de chambre » tout aussi inapte à subvenir à ses besoins alimentaires -

\footnotetext{
${ }^{14}$ « je criay beaucoup, à cause que cest animal si laid me faisoit peur » (p. 165).

${ }^{15}$ Confirmant l'étude de Jacques Gélis (voir note 11), Furetière indique sous la rubrique « Nourrir »: « une bonne mere doit elle-même nourrir son enfant. Les animaux nourrissent bien leurs petits » (Dictionnaire universel). Je souligne.

${ }^{16}$ La chute au jardin d'Eden est également subvertie dans l'histoire comique de Cyrano de Bergerac.

${ }^{17}$ Je propose les topoï « voleur de nourriture menacé de punition »; «personnage satisfait sa gourmandise/sa faim par la ruse ».
} 
et en l'occurrence scolaires - que le « maistre Singe » qui s'était introduit chez lui. Le narrateur s'attarde sur le régime bien frugal imposé aux élèves par Hortensius, principale cible de la satire dans les trois éditions de l'histoire comique. La «chiqueté » du pédant le pousse en effet à « espargner la plus grande partie de la pension [des écoliers], pour ne [les] nourrir que de regardeaux » (p. 171), en vue de se délecter à leurs dépens. Le narrateur, dont le pédant a remarqué le grand appétit, se lamente en ces termes : «Jamais Tantale ne fut si tenté aux Enfers par les pommes où il ne peut atteindre, que nous l'estions par ces bons morceaux où nous n'osions toucher » (p. 172). A la différence du bon maître Ponocrates qui prescrit une diète raisonnée à Gargantua pour faciliter le travail de l'esprit, Hortensius prêche l'abstinence pour mieux servir sa propre gourmandise. Comme justification pour son « vice insupportable » - et en guise de leçon aux écoliers -, le pédant régurgite des lieux communs, telle que la sentence attribuée à Cicéron : «Il ne faut manger que pour vivre, et non pas vivre pour manger » (p. 172). En revanche, fidèle au stéréotype du pédant glouton, Hortensius s'empiffre et s'enivre lors d'un repas qu'il organise en vue d'impressionner Fremonde dont il est tombé amoureux (p. 193-20).

Le récit de la vie au collège présente une série d'anecdotes montrant la subtilité de Francion pour assouvir sa faim, ou pour tirer vengeance du pédant qui se complaît à manger « ce qu'il avoit d'exquis » en face de ses écoliers pour les faire « enrager d'envie ». Cette configuration narrative n'est pas sans rappeler le roman picaresque, une influence du reste rendue explicite dans une remarque de Raymond, l'interlocuteur de Francion ${ }^{18}$. La première fourberie longuement décrite se focalise sur le larcin d'un pâté de lièvre que le père d'un des compagnons de Francion a offert à Hortensius. L'épisode a pour cadre l'étude du pédant, désigné comme un « cabinet autant sacré à Bacchus et à Ceres qu'aux Muses » (p. 176). Le narrateur précise à propos du mets délicat qu'Hortensius a dissimulé qu'il en fait « autant d'estat que de ses livres » (p. 176). Notons que le terme « pasté » est supprimé plusieurs fois dans la désignation de l'objet tantalien, ce qui permet le rapprochement du signifiant «lievre » et « livre». Une seconde anecdote met davantage en évidence l'origine étymologique commune de savoir et saveur (sapere). Cette fois, Francion pénètre dans l'étude d'Hortensius pour dérober une bouteille d'hypocras. Surpris par le pédant, il prétend chercher les œuvres d'Ovide et parvient bientôt à emporter à la fois le livre et la bouteille

\footnotetext{
${ }^{18}$ Raymond rapproche les «plaisantes aventures » de Francion des « actions basses », « infiniment agréables » des « gueux et des faquins [ajout, 1626 : comme de Guzman d'Alfarache et de Lazaril de Tormes] (p. 180). 
en la cachant «sous son manteau ». Le narrateur juxtapose objet de connaissance et nourriture dans un calembour : «Je n'avois que faire de l'Ovide, et eusse plutost affaire d'un os plein » (p. 193).

Comme le suggère l'opposition entre plénitude et vacuité, par le biais des références aux privations alimentaires, c'est le régime intellectuel prescrit dans les collèges qui est critiqué ${ }^{19}$. Faisant allusion aux « sornettes » et aux « mille grimauderies les plus pedantesques du monde » auxquelles sont exposés les écoliers, le narrateur constate ironiquement : « n’est-ce pas là une belle doctrine pour abreuver une jeune ame ${ }^{20}$ » (p. 183); ce à quoi il ajoute : " Mon Dieu, que les peres sont trompez, pensant avoir donnez leurs fils à des hommes qui les rempliront d'une bonne et profitable science » (p. 184). Le vice principal de l'enseignement de ces « barbares » est d'inciter les élèves à suivre leur exemple, privilégiant ainsi l'imitation servile ; ceux-ci n'apprennent qu'à cuisiner ou le plus souvent régurgiter des « capilotades a la pedantesque » (p. 184); autrement dit, d'indigestes ragoûts de sentences mal assaisonnées. Sorti du collège, Francion exprime un profond dégoût pour la maigre pitance et l'« innutrition » malsaine infligées à son esprit. Se souvenant des paroles qu'il a prononcées lors d'une pièce dirigée par le pédant - dans laquelle, entre autre niaiseries, on apporte un «boüillon et un consommé pour Jupiter» —, il remarque : « je pensay vomir tripes et boyaux, tant cela me fit mal au cœur» (p. 185).

L'évocation des études de Francion recouperait ainsi les implications du premier souvenir d'enfance : affamé de savoir, le protagoniste quitte le collège en quelque sorte « barbouillé » de la bouillie que lui ont présentée les piètres imitateurs qui lui servent de maîtres. Ayant purgé son entendement de ces «vieilles erreurs », il entend bien satisfaire sa soif de connaissance en le «remplis[sant] d'une meilleure doctrine » (p. 214).

\section{3. "Lascifs appetits" "}

Parallèlement à l'association récurrente entre fonction nutritive et acquisition du savoir qui caractérise l'épisode du collège, Sorel exploite un champ lexical confondant le domaine

\footnotetext{
19 "Nourrir signifie encore eslever, instruire" (Furetière). Les critiques de Sorel rejoignent celles de Montaigne dans l'essai "De l'institution des enfants" (I, 26). Wim de Vos envisage la nourriture comme «comparant» pour la rhétorique et l'éloquence (Le singe au miroir: Emprunt textuel et écriture savante dans les romans comiques de Charles Sorel, 1994, pp. 84-93).

${ }^{20}$ Les italiques dans les citations de Francion mettent en relief certains termes importants dans le réseau lexical considéré.
} 
alimentaire et la sexualité, autre lieu commun s'inscrivant dans la continuité d'une prédilection des écrivains de la Renaissance. Quittons le récit à la première personne retraçant la jeunesse de Francion pour considérer l'ouverture de l'histoire comique et sa conclusion.

Le premier livre présente l'échec de la tentative du protagoniste pour rejoindre l'ancienne prostituée Laurette après avoir éloigné son vieux mari, Valentin ; Francion a pu convaincre ce dernier que le rituel magique qu'il lui a prescrit pourrait lui « monstrer la voye de gouster les plus chers contentements du monde»(p. 69). Une suite de quiproquos occasionnée par des travestissements vient pourtant contrarier les attentes de Francion. Le motif du trompeur trompé se combine dans l'épisode avec celui de la substitution d'amant : tandis que le voleur Olivier prend place dans le lit de Laurette, le protagoniste tombe évanoui dans la cuve qui vient de servir aux ablutions de Valentin. Ce remplacement fortuit satisfait cependant la femme doublement infidèle. La confusion des registres lexicaux alimentaires et sexuels s'accentue : « celuy qu'elle avoit pris pour Francion luy avoit fait gouster des delices qu'elle n'eust pas possible gousté plus savoureus avec Francion mesme» (p. 76). Quant à Olivier, il se réjouit de pouvoir profiter d'un « si friand morceau » (p. 80).

Lorsque l'imbroglio se dénoue au lever du jour, les villageoises assemblées pour contempler ce qui pend entre les jambes du voleur déguisé en servante, qui se retrouve attaché à une fenêtre du château, profèrent quelques plaisanteries grivoises à propos de ce «gentil instrument» : «nous ne voulons pas avoir seulement part a un morceau : Mais le voulons avoir tout entier », s'exclame l'une d'entre elles. Un villageois répartit à cette « belle friande » : « Il est vray que vous estes la plus goulüe de toutes » (p. 84). Suite à d'autres équivoques liées au registre alimentaire, le passage se conclut en ces termes : « Ainsi plusieurs autres larderent les femmes de brocards en plus d'endroits que le plus sçavant Cuisinier de Paris ne larderoit une longe de veau » (p. 84).

L'appétit sexuel de Francion, frustré à l'ouverture de l'histoire comique, se voit en revanche pleinement assouvi dans l'épisode de la fête libertine qui prend place à la fin de la première édition. Les principaux personnages se retrouvent assemblés dans le château de Raymond qui annonce au protagoniste son intention de le faire « jouir de tous les délices dont [il] se pourr[a] aviser » (p. 308). Précisant qu'il a fait venir Laurette et cinq autres demoiselles, il poursuit en ces termes : «Il faut que nous facions tous ensemble une merveilleuse chere, l'inimitié que j'ay tesmoigné de vous porter, n'a esté que pour vous rendre maintenant plus savoureux les fruits de 
l'amitié que j'ay pour vous» (p. 308). Certes, en plus des «plaisirs de l'amour» auxquels le seigneur Bourguignon convie ses invités, sa générosité se traduit en premier lieu par un banquet, dont la profusion est proportionnelle aux plaisirs charnels qui s'ensuivent :

l'on vint dresser une longue table, qui fut incontinent chargée de tant de diverses sortes d'animaux, qu'il sembloit que l'on eut pris tous ceux de la terre, pour les manger là en un jour. Quant l'on eut estourdy la plus grosse faim, Raymond dit à chacun qu'il faloit [...] se resoudre a faire la desbauche, la plus grande dont il eut jamais esté parlé (p. 313).

Selon la tradition du banquet, Francion et Raymond ne manquent pas de mêler au plaisir des sens celui de la conversation ${ }^{21}$. La nuit venue, on présente en guise de souper à cette lascive assemblée « une magnifique collation », comprenant « force viandes des plus exquises desquelles ceux qui avoient faim pûrent se rassasier ». Les confitures sont également au menu : « [elles] étoient en si grande abondance, que chacun en ayant remply son corps et ses pochettes, il en demeura beaucoup ». Le tout, accompagné « des meilleurs vins du monde » (p. 316-17).

Ainsi, pendant sept jours, Francion et ses compagnons « se donnerent tout le bon temps que l'on peut s'imaginer » (p. 323), de quoi assouvir provisoirement les appétences du protagoniste qui déclare avoir « plus de desirs qu'il n’y a de grains de sable en la mer » (p. 315). Cependant, la narration de cet épisode marqué par l'opulence et la satiété se conclut sur l'arrivée inattendue de Valentin qui - suivant un topos privilégié par les nouvelles de la Renaissance - entend tirer vengeance de Laurette dont il a découvert la tromperie. «Vomissant son fiel », ce mari dépité la menace de privation : «Tu as icy gousté a cœur saoul des plaisirs avec les hommes [...]. Mais desormais, je te feray jeusner maugré que tu en ayes, et tu n'auras plus de moy ta pitance ordinaire » (p. 325). Pour calmer le courroux du vieux mari, on lui fait « avaler sa tristesse, avec plusieurs verres de vin » (p. 326).

L’arrivée incongrue de Valentin, personnage associé aux «opinions vulgaires » dès l'ouverture de l'histoire comique, met davantage en évidence l'exigence de raffinement qui préside à cette festivité libertine se déroulant en huis-clos. Réservé aux «âmes généreuses » selon l'inscription placée sur la porte de la grande salle, l'événement s'oppose radicalement aux noces paysannes, qui sont célébrées dans la parcimonie, mais au cours desquelles la notion d'excès est déplacée : l'appétit des «brutaux »s'y voit ridiculisé en raison de l'effet de la «composition

${ }^{21}$ Voir Michèle Rosellini, « La fête libertine du Francion : du festin au banquet philosophique », 2000, pp. 67-82.

Topiques, Études satoriennes, 5, 2020, Le Boire et le manger, https://journals.uvic.ca/index.php/sator/index 
laxative » que Francion place dans le potage. Par ailleurs, le protagoniste insiste pendant l'orgie sur la distinction entre les « Païsans » et la sélecte compagnie à laquelle il s'adresse, au niveau de l'acte sexuel : les « brutes » se contentent de « saouler leur appetit stupide » (p. 321), sans pouvoir accéder aux « transports d'esprit » de ceux, qui comme lui et ses compagnons, y apportent quelque chose de « divin et de céleste » (p. 322).

\section{Gourmandise onirique}

Le rapprochement nourriture/sexualité ainsi que la dichotomie abondance/privation soustendent l'agencement narratif du long épisode du rêve sur lequel s'ouvre le troisième livre. Récit que son destinataire, Raymond, estime « extremement delicieux a entendre » (p. 140) et promet d'interpréter par la suite « entre la poire et le fromage » (p. 155). Au début de ce parcours onirique extravagant, le protagoniste a l'occasion de se «rassasier» en faisant preuve d'inventivité. Annonçant le savoir-faire dont il fait preuve au collège, il constate que « le désir de contenter son ventre est un maistre en toutes sortes de sciences et d'arts » (p. 141). Francion utilise le membre viril dont il se vante d'être bien « fourni » pour boucher le trou de la cuve dans laquelle il flotte ; il parvient ainsi à saisir à pleines mains les concombres, les melons, les cervelas et les saucisses qui tombent du ciel. Alors qu'il vient de se « rempli[r] le ventre de saucisses, qui [lui] sembloient un délicieux manger », ce sont d'autres objets de convoitise qui attirent l'attention du protagoniste à la dérive : «En estant tout rassasié je m’amusay a contempler une belle Isle [...] où je voyois des nourritures bien plus exquises que celles dont je m'estois saoulé » (p. 141). Mais c'est en vain, cette fois, que Francion tente de les atteindre ; elles lui restent inaccessibles, même lorsqu'il se jette «à corps perdu» dans le lac: «Ceste terre que je croyois estre fort proche estoit fort esloignée, et si elle se reculoit à mesure que j'avançois » (p. 141). Le récit se poursuit en modulant l'évocation de frustrations de nature sexuelle; limitons-nous à deux occurrences narratives relatives aux « appas » de Laurette. Dans une première séquence, celle-ci s'évanouit entre les bras de Francion alors qu'il tente de l'embrasser (p. 151); nouvelle déconvenue pour le protagoniste lorsqu'il s'empresse plus tard d'aller toucher ce corps qu'il convoite : le voyant en "grande peine », son guide lui apprend que Laurette « estoit enfermée d'un estuy de verre » (p. 153). De quoi rivaliser avec les supplices de Tantale!

Le paysage fantasmagorique dans lequel circule le protagoniste juxtapose ou confond nourriture, breuvage, et composantes corporelles/sexuelles. Après avoir rencontré des monstres 
qui habitent un palais bâti « avec toute sorte de choses bonnes a manger » (p. 145), Francion tombe dans « un lieu couvert de jeune tetons collez ensemble deux à deux » (p. 149) sur lesquels il se vautre. Par ailleurs, alors que « la matiere des ames » ayant la douceur d'un lait d'ânesse remplit un bassin de cristal (p. 143), des pucelages de femmes, ressemblant à une « liqueur », sont exposés dans un temple consacré au Dieu d'Amour (p. 151). Le motif de l'anthropophagie s'insinue dans le mélange des registres. Francion échappe de justesse à la menace d'une «majestueuse Dame » qui partage le repas de monstres infligeant des supplices à des hommes nus. Montrant Francion du doigt, « elle leur commanda de prendre [s]on cœur et leur asseura que ce luy [à la dame] seroit une viande très savoureuse $»($ p. 147).

Cet exemple de la victimisation subie par le protagoniste dans le récit onirique nous amène à considérer un déplacement subversif du processus d'ingestion. À plusieurs reprises, Francion ingurgite un breuvage contre son gré, voire sous la contrainte. En premier lieu, il est précipité au fond du bassin de cristal ; la « liqueur » qu'il contient se révèle être une « certaine fumée espaisse » (p. 144). Par la suite, une « belle Dame » lui met un entonnoir dans la bouche, lui faisait espérer l'administration d'une « liqueur délicieuse » :

J'ouvrois desja le gosier plus large que celuy de ce Chantre qui avalla une souris en beuvant, lorsque s'estant un peu relevée, elle pissa plus d'une pinte d'urine, mesure de sainct Denis, qu'elle me fit engorger (p. 149).

L'élément liquide s'avère à nouveau néfaste lorsque Francion se retrouve métamorphosé en monstre, sous l'incitation d'une « ancienne matrone » qui lui laisse envisager des «perfections infinies » : « je n'eus pas si tost beu plein ma main, que me mirant dedans l'eau, je vy que j'avois la plus laide forme que l'on puisse figurer » (p. 145). Francion apprend par la suite que l'eau avait été altérée par un monstre qui s'y était « lavé la teste et y avoit laisse sa laideur» (p. 147) ${ }^{22}$. Le phénomène de contamination présenté par l'absorption du liquide dans le rêve renvoie ainsi, sur

\footnotetext{
${ }^{22}$ Les occurrences venant d'être présentées combinent deux topoï : «une femme séductrice/perfide trompe un personnage »; « un personnage naïf est puni par sa convoitise ». Par ailleurs, cette récurrence narrative pourrait être envisagée comme une variante du motif de l'empoisonnement.
}

Topiques, Études satoriennes, 5, 2020, Le Boire et le manger, https://journals.uvic.ca/index.php/sator/index 
le plan discursif, au malaise associé au lait de la nourrice, susceptible de transmettre au nourrisson des humeurs vulgaires, comme nous l'avons remarqué23.

\section{5. "De la modération"}

Réceptacle de l'abondante nourriture tombée du ciel, la cuve dans laquelle Francion part à la dérive au début du rêve est associée au seul moment de pleine satisfaction du protagoniste avant qu'il ne soit « englouty des flots » (p. 138). Or, sa nacelle est explicitement rapprochée de celle où il tombe évanoui au cours de son entrée en scène : « parce que mon esprit estoit remply de la memoire des choses qui m'arriverent hier, il me semble que j'estois encore dans une cuve [...]. Je flottois là dedans sur un grand lac» (p. 140). On se souvient des conséquences presque fatales de cette chute, dues à une excessive ingestion : "[Francion] n'eut pas le soin de s'empescher d'avaller un grande quantité d'eau dont il pensa estre noyé » (p. 74).

La position ridicule dans laquelle Francion est placé au début de l'histoire comique trouve un parallèle dans une situation évoquée par Raymond. Encourageant ses invités à boire, il fait allusion à la légende de la mort de Georges Platagenêt, réputé bon buveur :

souhaittons de mourir comme George, Comte de Clarence, qui se voyant contraint par le jugement du Roy d'Angleterre de quitter la vie, se fit mettre dans un tonneau plein de vin, dont il beut tant qu'il en creva (p. 318).

Le contraste burlesque suggéré par le rapprochement de ces deux situations nous amène à considérer le rapport de Francion au vin, breuvage primordial dont les vertus font l'objet de commentaires diététiques et d'une réflexion morale à l'époque.

Comme point de départ, il convient de considérer le ton de bravade sur lequel le protagoniste tire les conséquences de sa naissance, le jour de la fête des rois :

L'on beut si plantureusement a ma santé par tout le logis qu'il y parut bien aux tonneaux de nostre cave. Maintenant il ne faut pas s'estonner si je boy bien, car c'est que me voyant en aage competant, je veux faire raison a loyale mesure a tous ceux qui m'appellerent des ce temps là au combat du verre, et je pense que je les y vaincray. (p. 164)

\footnotetext{
${ }^{23}$ Voir la remarque de Jacques Gélis sur les conséquences de la « transfusion » du « lait mercenaire » (note 11). Pour une élaboration de cette occurrence par rapport à la chute de Francion dans la cuve que Valentin aurait contaminée de son impuissance, voir Martine Debaisieux, Le Procès du Roman, pp. 80-88).
} 
Il s'avère cependant que l'allusion à cette vaillance et à cette supériorité face à la bouteille est contredite, lors de deux échanges sur le «divin breuvage ». Le premier correspond aux toutes premières paroles de Francion à l'ouverture de l'histoire comique, au moment d'une renaissance symbolique. Alors qu'il se remet de son évanouissement dans la cuve remplie d'eau, il reçoit la visite d'un chirurgien dans l'hôtellerie où il a trouvé refuge. Ce dernier s'oppose d'emblée à ce que le convalescent boive le vin qu'on lui apporte :

Francion ayant ouy cet avis si rigoureux dit: Ho Monsieur, ne me privez point de ce divin breuvage, je vous en prie, c'est luy qui est le seul soutien de mon corps, toutes les viandes ne sont rien au prix (p. 87).

Un jeu de contradictions se met alors en place : à la suite de propos visant à convaincre le chirurgien de lever son interdiction, Francion indique qu'il ne faisait que plaisanter et qu'il s'abstiendra bien de boire. Se présentant maintenant comme défenseur d'une éthique corporelle fondée sur le discours moral classique, il conclut en ces termes : «C'est a faire aux ames basses [...] a ne pouvoir de telle sorte commander sur eux mesmes, qu'ils ne sçachent restraindre leurs apetits et leurs envies » (p. 88). Ironiquement, tout en faisant allusion aux prescriptions diététiques des Anciens, le chirurgien reconnaît qu'il serait quant à lui incapable de suivre les principes de modération qu'implique la seconde vertu cardinale :

Vostre temperance est remarquable [...]. Je n'ay pas les ressors de l'ame si fermes qu'ils puissent ainsi commander a mon corps, car je vous asseure bien que quand Hypocrate mesme m'auroit dit que l'usage du vin me seroit nuisible, je ne m'en priverois pas, et que quand l'on me mettroit aupres d'une fontaine d'eau, je ne lairois pas [= manquer de $]$ de mourir de soif (p. 88).

C'est dans l'épisode de l'orgie que l'on trouve une seconde référence à la position de Francion à l'égard du vin ${ }^{24}$. Elle s'insère dans l'échange au cours duquel son hôte fait allusion à la mort exemplaire du comte de Clarence : «Raymond qui se plaisoit fort au combat du verre, fit apporter des meilleurs vins du monde [...]. Il n'est rien de pareil à ce breuvage, dit il, il emplit d'une certaines divinité ceux qui l'avallent» (p. 318). Ayant fait l'apologie de cette divine boisson, qui apporte courage et vaillance, il invite Francion à s'approcher du tonneau après s'être exclamé

\footnotetext{
${ }^{24} \mathrm{~L}$ 'expression de la retenue de Francion par rapport au vin le rapproche de Théophile qui remarque dans son histoire comique Première journée (1623): «le vin m’a souvent réjoui, mais jamais enivré » (1999, p. 15). Cette « disposition » distingue le narrateur de son compagnon Sydias — stéréotype du pédant glouton comme Hortensius — qu'il retrouve ivre-mort dans l'hôtellerie.
}

Topiques, Études satoriennes, 5, 2020, Le Boire et le manger, https://journals.uvic.ca/index.php/sator/index 
« beuvons, beuvons eternellement ». Contrairement à la prérogative prétendûment conférée par les circonstances de sa naissance, le protagoniste refuse le «combat du verre » proposé par son compagnon. Dans sa répartie, Francion se présente à nouveau en adepte de la modération, guidé cette fois par la vertu de prudence, qui lui indique une option préférentielle :

Je n'en feray rien, respondit-il, j'ayme mieux user mes forces en me jouant avec Laurette, qu'en me jouant avec Bacchus. Si j'en prenois trop, tout mon corps seroit brutalement assoupy, et ne pourroit plus prendre avec les femmes qu'un plaisir lent, et j'ose bien dire douloureux (p. 338).

\section{De Bacchus aux Nä̈ades?}

À l'issue de cette semaine de débauche où il jouit de Laurette « tant qu'il avoit voulu », Francion s'apprête à rejoindre Nays, dont le portrait exposé dans la chambre qu'il occupe chez Raymond l'a séduit. Suivant la prescription de ses médecins, la belle veuve italienne est allée « boire certaines eaux » pour remédier à une « indisposition » (p. 324); un déplacement qui accentuerait son association aux « Nayades » que contemplait Francion collégien ${ }^{25}$. C'est donc cette fois sous le signe de l'eau, plus précisément d'une eau curative, que s'annonce la quête qui fait l'objet des seconde et troisième éditions : « je m'en iray aux eaux pour tascher d'y rencontrer Nays » (p. 324). Alors que l'évocation de la naissance dans la première édition insinue que le contenu des tonneaux de vin est proportionnel à la consommation des invités, l'eau thermale, coulant de source, échapperait à cette diminution ou à ce tarissement potentiel. Notons que le motif de la pénurie trouverait un remède encore plus concluant dans la conception d'une «fontaine perpétuelle » suggérée dans Le Berger extravagant, histoire comique présentée comme « Seconde Partie » de Francion $^{26}$.

Si la vénération de Francion envers le portrait de Nays se conforme à la topique du roman sentimental, elle n'empêche pas certains infidèles détours sur le chemin vers l'Italie. Ainsi, suite aux récits d'imbroglios amoureux qui retardent la conclusion de l'histoire comique et se compliqueront nettement dans l'ajout d'un douzième livre, Nays est consciente que Francion « avoit falsifié ses affections, et corrompu l'amour » (p. 497). Le narrateur présente un personnage

\footnotetext{
${ }^{25}$ Influencé par ses lectures, Francion s'imagine qu'il y a « des Nayades aux fontaines » (p. 213).

${ }^{26}$ Le protagoniste Lysis imagine un dispositif pour métamorphoser son serviteur Carmelin en fontaine « artificielle, portative et eternelle». Olivier Roux retrouve cette "obsession sorélienne» dans La Solitude et l'amour philosophique de Cléomède et dans La Science universelle, où Sorel propose deux dispositifs permettant une « circulation perpétuelle» de l'eau (Charles Sorel : La figure, la ligne et l'invention de l'auteur, 2014, pp. 219-22).
} 
repentant, lorsqu'il s'adresse à ses compagnons la veille de ses noces. Dans la dernière version du texte, Francion déclare que « desormais il tascheroit d'estre plus sage que par le passé, et qu'il croyoit qu'ayant espousé Nays il seroit arrivé a bon port [...]» (p. 526). Un soupçon face à cette déclaration, déjà éveillé par deux nuances absentes de l'édition précédente (« il tascheroit »; « il croyoit »), est renforcé par un ajout: « Il taschoit de persuader aux autres [ses compagnons] de se retirer ainsi de leur vie desbauchée le plus tost qu'ils pourroient, afin de ne plus servir de mauvais exemple » (p. 526 ; je souligne).

Les allusions au mariage de Francion à Rome, en compagnie de quelques compagnons, introduisent certaines équivoques relatives au domaine alimentaire. L'édition de 1627 fait précéder l'événement d'un souper de fiançailles chez Nays où la référence à « deux perdreaux » et « quatre pigeonneaux » n'est que prétexte à une « dispute de Sophiste » menée par Hortensius, qui cherche à montrer son «subtil esprit» pour régler à son avantage le partage de ce repas, peu élaboré semblerait-il (p. 459). Supprimées dans la dernière édition, les toutes dernières paroles du personnage dans cette séquence narrative - et dans l'histoire comique - s'avèrent en revanche corrosives :

Francion luy demanda ce qui luy sembloit de Nays, et s'il ne l'estimoit pas heureux d'avoir une si belle Maistresse. Hortensius qui n'avoit pas assez de prudence pour celer ce qu'il pensoit, luy respondit que les secondes nopces n'avoient rien de meilleur que les viandes reschaufées (p. 461).

Cette comparaison alimentaire surprenante vient renforcer la réprobation de la « loy » du mariage exprimée par Francion lui-même au cours de l'orgie : ces « fascheux liens » sont à l'origine de « tant des maux » (p. 315) 27.

Le douzième livre ajouté à la dernière édition de Francion remplace le souper de fiançailles par une simple allusion à un dîner, exempt de toute référence au boire et au manger. Il sert uniquement de cadre à une conversation où les compagnons, finalement réunis, font le bilan de leurs « avantures » (p. 524). L'accomplissement du projet de mariage est soulevé à la fin de ces propos, non par Francion, mais par Dorini, qui suggère de ne plus « tant [le] faire trainer »; « l'on envoya donc querir un Prestre et ils furents fiancez tout a l'heure, et il fut arresté [que Francion et

\footnotetext{
${ }^{27} \mathrm{Il}$ s'agit d'une longue réplique à la maquerelle Agathe : « je voy bien que si tout le monde vous ressembloit, l'on ne sçauroit ce que c'est que de mariage, et l'on n'en observeroit jamais la Loy : vous dites vray, respondit Francion [...] » (p. 315).
} 
Nays] seroient mariez le lendemain » (p. 525). En contraste avec la fête libertine bien arrosée à la fin de la première édition dix ans auparavant, et avec les noces paysannes diarrhéiques, la célébration du mariage de Francion est marquée par la retenue et la sobriété. La brève mention de l'événement - similaire dans les deux éditions à l'exception du dernier mot - se passe de toute allusion au boire et au manger : « ce n'est pas la coutume que l'on assemble beaucoup de monde au mariage d'une vefve ni que l'on y fasse beaucoup de magnificences » [dernier mot remplacé par «resjoüyssance», 1626; (p. $527 /$ p. 461)]. A ce manque de convivialité et d'opulence correspond une nouvelle image du protagoniste : «Francion se voyant obligé de ne plus vivre en garçon prit dès lors une humeur si grave et si serieuse que l'on n'eust pas dit que c'eust esté luymesme » (p. 527). L’humeur de ce séducteur (soi-disant) réformé serait ainsi en harmonie avec un plaisir conjugal qui se doit d'être « retenu, sérieux et mêlé de quelque sévérité » si l'on se fie à l'essai de Montaigne « De la modération ${ }^{28} »$.

Et pourtant, en dépit de la mention rapide de cette sobre cérémonie et de la métamorphose d'un protagoniste dont les aspirations sporadiques à la tempérance se verraient outrepassées par les « fascheux liens » conjugaux, Sorel ne ferme pas complètement la porte aux « réjoüyssances » et à la satiété. En effet, les plus proches compagnons de Francion ne sont guère enclins à suivre la voie de la retenue et de la modération : «Quand a Raymond et du Buisson, quelque remonstrance [que Francion] leur pust faire, ils employerent encore le reste du temps qu'ils vouloient passer dans Rome, a se saouler des plaisirs du monde» (p. 527). Transporter dans la capitale italienne Raymond, capiteux personnage resté fidèle à Bacchus, pour le placer aux côtés d'un protagoniste « corrigé » de manière peu convaincante dans les révisions du texte - et reconnaissant sa sensibilité au « mauvais exemple » -, n’était-ce pas permettre aux effluves de la débauche libertine de franchir les frontières des éditions successives, et de brouiller les pistes menant au scénario final?

Certes, la conjecture d'un allègre revirement pour Francion face à ses noces frugales et à la « grave » tempérance qu'elles représentent reste inscrite entre les lignes de cette conclusion « postiche » et sur le « palimpseste » des variantes ${ }^{29}$. En revanche, pour conclure plus directement sur le domaine du boire en proposant la réconciliation de deux éléments emblématiques qui ont

\footnotetext{
${ }^{28}$ Essais, I, 30 (1965, p. 296). Sorel consacre une section de La Bibliothèque françoise aux Essais, dont il apprécie la « hardiesse » et la « vigueur » (p. 89).

29 J'emprunte ces deux expressions à Patrick Dandrey (2001).
} 
guidé la dernière partie de notre parcours, revenons à la fin du premier livre. Dans cet épiode, Francion montre à Valentin quelques secrets de « magie naturelle » qu'il a appris en fréquentant les alchimistes. Or, il s'avère que le protagoniste est capable de « transmuer l'eau en vin » (p. 99) 30 ; raillerie d'un cru libertin d'autant plus significative si l'on se souvient que, dans la liturgie chrétienne, les Noces de Cana coïncident avec la fête de l'Épiphanie, pendant laquelle Sorel situe la naissance de son protagoniste.

\section{Bibliographie}

\section{Euvres et sources}

FURETIÈRE, Antoine, Dictionnaire universel, Genève, Slatkine Reprints, 1972. (Réimpression de l'édition de La Haye-Rotterdam, 1690).

MONTAIGNE, Michel de, Essais, Livre Premier, Ed. Pierre Michel, Paris, Gallimard, 1965.

SOREL, Charles, La Bibliothèque françoise, 2nd édition, 1667, Genève, Slatkine Reprints, 1970.

— De la Perfection de l'homme, Paris, Robert Le Nain, 1655.

- Histoire comique de Francion, In Romanciers du XVIIe siècle, éd. Antoine Adam, Paris, Gallimard (Bibliothèque de la Pléiade), 1958.

VIAU, Théophile de, Première Journée. CEuvres complètes (II), éd. Guido Saba, Paris, Honoré Champion, 1999. pp. 9-30.

\section{Études critiques}

ASSAF, Francis, « Francion, une étude carnavalesque », Littératures classiques, 41, 2001, pp. 8595.

\footnotetext{
${ }^{30}$ Valentin voit un miracle dans ces effets de « magie naturelle ». Fausta Garavini metionne ce passage où la critique de la crédulité atteint la foi catholique (1975, p. 59). Jole Morgante repère dans d'autres scènes l'« audace sacrilège » de Sorel par rapport au dogme chrétien (2001, pp. 14-30). Voir aussi, François Friche, Entre Terre et Ciel: romans comiques et mystère de l'Incarnation (2017).
} 
BAKHTINE, Mikhaïl, L'œuvre de François Rabelais et la culture populaire au Moyen Age et sous la Renaissance, Paris, Gallimard, 1970.

DANDREY, Patrick, Le premier Francion de Charles Sorel, ou le " jeu du roman », Paris, Klincksieck, 2001.

DEBAISIEUX, Martine, « Francion ou la libre pensée travestie », Neophilologus, 72, 1988, pp. 191-99.

- Le Procès du Roman: Ecriture et contrefaçon chez Charles Sorel, Saratoga, Anma Libri, Stanford French and Italian Studies, 1989. Republication, Éditions Paradigme, Orléans, France, 2000.

DE VOS, Wim, Le singe au miroir : Emprunt textuel et écriture savante dans les romans comiques de Charles Sorel, Tübingen, Gunter Narr Verlag, 1994.

FLANDRIN, Jean-Louis, "Alimentation et médecine. Histoire de l'alimentation occidentale » http://www.lemangeur-ocha.com/texte/alimentation-et-medecine/; mis en ligne 28/04/2003

FRICHE, François, Entre Terre et Ciel : Romans comiques et mystère de l'Incarnation (16201660), Paris, Hermann, 2017.

GODARD DE DONVILLE, Louise, Le Libertin des origines à 1665 : un produit des apologètes, Paris-Seattle-Tübingen, Biblio 17 (51), 1989.

GARAVINI, Fausta, «Francion rivisitato : Diacronia d'una struttura », Saggie et ricerche di letteratura francese, XIV, 1975, pp. 39-107

- La Maison des Jeux : Science du roman et roman de la science au XVII siècle, Paris, Champion, 1998.

GÉLIS, Jacques, « L'individualisation de l'enfant », Histoire de la vie privée, 3, De la Renaissance aux Lumières, Ed. Philippe Ariès et Georges Duby, Paris, Seuil, 1986, pp. 311-31.

HODGSON, Richard G., « De la 'comédie humaine' à la 'perfection de l'homme' : Charles Sorel moraliste », in Charles Sorel polygraphe, éd. Emmanuel Bury et Eric Van der Schueren, Laval, Presses de l’Université Laval, 2006, pp. 19-30.

HOWELLS, Robin, Carnival to Classicism: The Comic Novels of Charles Sorel, Paris-SeattleTübingen, Biblio 17 (48), 1989.

JEANNERET, Michel, Des Mets et des mots : Banquets et propos de table à la Renaissance, Paris, Corti, 1987. 
MORGANTE, Jole, « La réécriture de la première partie du Francion : techniques d'écriture libertine », Littératures Classiques, 41, 2001, pp. 14-30.

ROSELLINI, Michèle, "La fête libertine du Francion : du festin au banquet philosophique ", Cahiers textuels, « Charles Sorel, Histoire comique de Francion », 22, 2000, pp. 67-82.

ROUX, Olivier, Charles Sorel : La figure, la ligne et l'invention de l'auteur, Paris, Champion, 2014. 